\title{
The Effects of Hyperbaric Oxygen Therapy in Patients with Central Retinal Artery Occlusion: A Retrospective Study, Systematic Review, and Meta-analysis
}

\author{
Luca Rosignoli, Edward R. Chu, John E. Carter, Daniel A. Johnson, Jeong-Hyeon Sohn, Sepehr Bahadorani \\ Department of Ophthalmology, University of Texas Health, San Antonio, TX, USA
}

Purpose: Central retinal artery occlusion (CRAO) is a vision-threatening condition with a potentially poor visual prognosis. Many different treatment modalities are suggested but controversy remains regarding effectiveness of these treatments. The purpose of this study is to perform a systematic review and meta-analysis in addition to analyzing retrospective data at our own tertiary care center regarding effectiveness of hyperbaric oxygen therapy (HBOT) in treatment of CRAO.

Methods: The PubMed, Scopus, and the Cochrane Library are searched from the date of database inception to September 2021 to conduct a review based on the PRISMA (preferred reporting items for systematic review and meta-analysis), evaluating the role of HBOT in visual recovery of CRAO patients. In addition, a retrospective chart review of patients clinically diagnosed with CRAO at our university-based hospital (University of Texas Health, San Antonio, TX, USA) from year 2011 to 2021 was conducted.

Results: After a review of 376 articles, three articles met the inclusion criteria for meta-analysis, where a total of 207 patients received HBOT versus 89 patients that did not receive any form of oxygen therapy. Analysis of these results demonstrate that HBOT in CRAO patients does not enhance the final visual outcome $(p=0.83)$. Similar conclusion was also drawn from retrospective analysis of 48 patients (15 HBOT versus 33 controls) at our tertiary care center, where no visual benefit was observed in the HBOT group.

Conclusions: HBOT does not appear to improve final visual outcome and concerns remain regarding adverse reactions such as barotrauma and generalized seizures. Large, randomized studies are required for further understanding of the role of HBOT in treatment of CRAO.

Key Words: Hyperbaric oxygenation, Meta-analysis, Retinal artery occlusion

Received: August 20, 2021 Final revision: October 29, 2021 Accepted: November 5, 2021

Corresponding Author: Sepehr Bahadorani, MD, PhD. Department of Ophthalmology, University of Texas Health, 7703 Floyd Curl Drive, San Antonio, TX 78229, USA. Tel: 1-210-567-8401, Fax: 1-210-567-8413,

E-mail: bahadorani@uthscsa.edu
Central retinal artery occlusion (CRAO) is an ophthalmic emergency and yet, no standardized management protocol exists for this disease. Over years, multiple CRAO management strategies have been proposed, including ocular massage, intraocular pressure lowering therapies, administration of thrombolytic agents, and supplemental oxygenation such as hyperbaric oxygen therapy (HBOT). 
The goal of these treatments is to increase blood flow and oxygenation to the ischemic inner retina. Nevertheless, none has consistently been shown to improve visual outcome following CRAO [1-5].

Similarly, HBOT has been employed as a potential management strategy in CRAO. It has been proposed that HBOT may limit ischemic retinal damage between the onset of CRAO and retinal artery recanalization, which typically occurs within 72 hours [6]. However, controversy remains regarding the effectiveness of this treatment modality in final visual outcome. Hence, the goal of this study is to understand the potential visual benefits of HBOT in the treatment of CRAO by performing a systematic review and to compare meta-analysis results to outcomes at our own clinical setting.

\section{Materials and Methods}

This study was approved by the institutional review board of University of Texas Health. Due to the retrospective nature of the study, written informed consent was waived.

We have conducted this study in accordance with widely accepted PRISMA (preferred reporting items for systematic review and meta-analysis) guidelines [7], with each stage of the study carried out by two researchers independently (SB and LR).

\section{Search strategy and selection criteria}

We have searched the PubMed, Scopus, and the Cochrane Library from the date of database inception to September 2021, searching for the following keywords: "oxygen central retinal artery occlusion." Inclusion criteria included patients with CRAO treated with HBOT and primary outcome assessment with initial and final visual acuity (mean \pm standard deviation). Exclusion criteria included non-English articles, case reports, reviews, and noncomparative data. The title and/or abstracts were reviewed by SB and LR independently, after which the full text of selected articles was reviewed.

\section{Data extraction and quality assessment}

Information on the first author, publication date, study design, sample size, initial and final visual outcome, mean elapsed time between symptom onset and treatment, and inclusion of adjunct therapies is extracted from selected studies. Egger's and Begg's quality assessment tests were not performed due to a small number of selected studies.

\section{Statistical analysis}

The RevMan ver. 5.4 software (Cochrane Collaboration, Copenhagen, Denmark) was used to assess continuous variable outcomes of visual acuity using the weighted

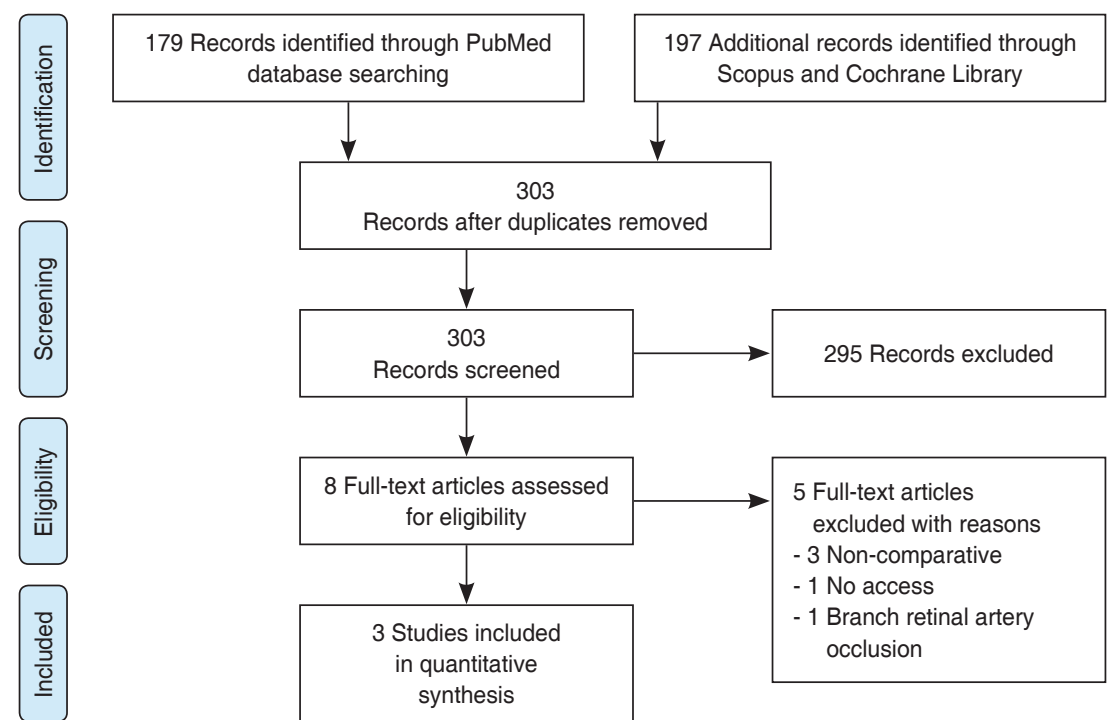

Fig. 1. Flow chart of literature search using PRISMA (preferred reporting items for systematic review and meta-analysis). 
mean difference, and the overall effect size with $95 \%$ confidence interval was calculated using a random model. The heterogeneity of studies was accessed using the chi-square test ( $p<0.10$ represents significant heterogeneity), and a forest plot was created using the RevMan ver. 5.4 software ( $p<0.05$ represents statistically significant).

\section{Retrospective analysis}

To compare the results from meta-analysis to outcomes under our own clinical settings, we have conducted a retrospective chart review of 121 patients presenting with CRAO at Univserity of Texas Health, San Antonio, TX, USA from year 2011 to 2021 . Of these 121 patients, 48 have met our inclusion criteria, where 15 underwent HBOT while the other 33 patients did not receive any form of oxygen therapy. Final outcomes included change in visual acuity (VA) from presentation to the final follow-up visit and incidence of neovascular glaucoma (NVG) between the two groups. Statistical significance for the VA and NVG is analyzed using Student $t$-test and chi-square test, respectively. Patients with unknown time of symptom onset, patent cilioretinal artery on exam, alternative diagnoses, or those that were lost to follow-up were excluded from our retrospective study.

The protocol for HBOT is based on the US Navy diving manual [8], with hyperbaric therapy being discontinued if no improvement in VA was noted during initial treatment. If the patient noted a recovery in vision, however, additional daily hyperbaric treatments using the same protocol were administered until no further improvement was obtained.

\section{Results}

\section{Meta-analysis outcome}

As illustrated in Fig. 1 flow chart, among 376 articles screened for eligibility, three articles met the final inclusion criteria for the qualitative assessment role of HBOT in CRAO patients. The relevant information from each article is summarized in Table 1 [9-11]. The same table also includes information from retrospective analysis of results at our own university hospital ("Current study"). Comparison of results for the selected articles demonstrate that HBOT

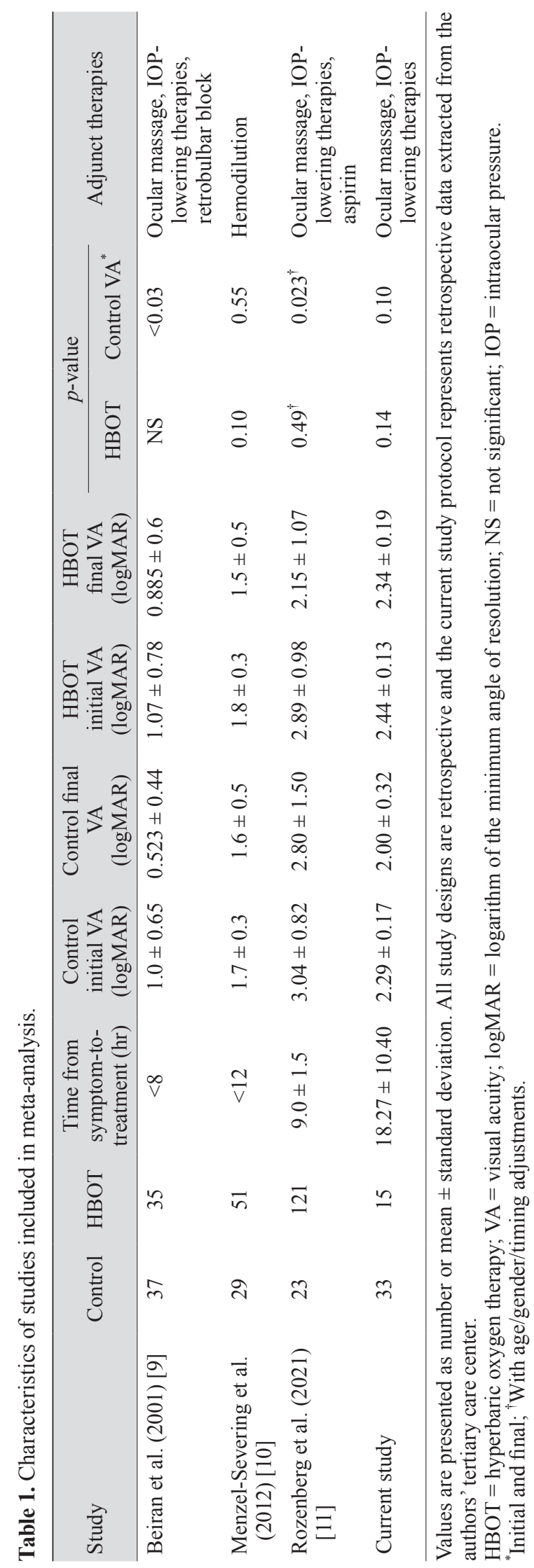


$(\mathrm{n}=207)$ does not provide a significant improvement in final VA $(p=0.83)$ over the control $(\mathrm{n}=89)$ group, as demonstrated in Fig. 2A [9-11]. Likewise, comparison of changes in the VA from initial to final presentation yields similar results, with no statistical significance $(p=0.52)$ between the HBOT and control groups (Fig. 2B) [9-11].

It is worth noting that the heterogeneity test for the se- lected studies (Fig. 2) yielded a $p$-value of $<0.1$, indicating inconsistency in the reported outcomes [9-11]. This discrepancy is most likely attributed to large differences in the baseline visual acuities in different studies, which, despite not significantly different at baseline within each study, might attribute to a source of meta-analysis conclusion bias due to large variations across studies. At the same

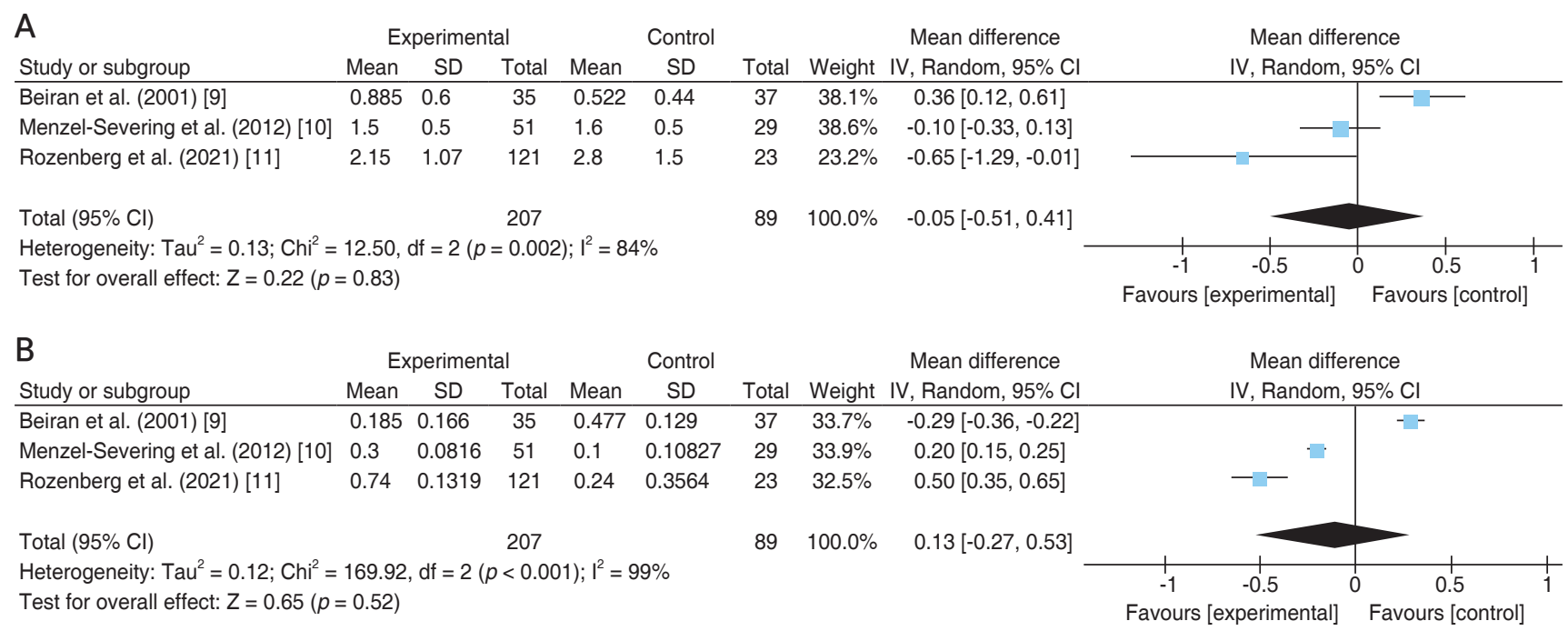

Fig. 2. Forest plot of the effects of hyperbaric oxygen therapy ("Experimental") versus no oxygen therapy ("Control") on visual acuity of patients with central retinal artery occlusion. Mean difference of (A) final visual acuity and (B) visual acuity change from initial to final presentation. $\mathrm{SD}=$ standard deviation; IV = inverse variance; Random = random-effect model; $\mathrm{CI}=$ confidence interval.

Table 2. Summary of the demographic information, risk factors, and supplementary treatments received in patients with central retinal artery occlusion

\begin{tabular}{lccc}
\hline Variable & Hyperbarics $(\mathrm{n}=15)$ & No hyperbarics $(\mathrm{n}=33)$ & $p$-value \\
\hline Age (yr) & 69.3 & 62.3 & $0.950^{*}$ \\
Male : female & $2: 3$ & $2: 1$ & $0.082^{\dagger}$ \\
Relative afferent pupillary defect & $14(93.3)$ & $24(72.7)$ & $0.103^{\dagger}$ \\
Neovascular glaucoma & $3(20.0)$ & $5(15.2)$ & $0.676^{\dagger}$ \\
Risk factors & & & $29(87.9)$ \\
Hypertension & $14(93.3)$ & $12(36.4)$ & $0.57^{\dagger}$ \\
Type 2 diabetes mellitus & $3(20.0)$ & $23(69.7)$ & $0.051^{\dagger}$ \\
Current or past tobacco & $6(40.0)$ & & $0.051^{\dagger}$ \\
Supplementary treatments & & $8(24.2)$ & $2(6.0)$ \\
Timolol 0.5\% / dorzolamide 2\% & $13(86.7)$ & $2.29 \pm 0.17$ & $<0.05^{\dagger}$ \\
Anterior chamber paracentesis & $2(13.3)$ & $2.00 \pm 0.32$ & $0.40^{\dagger}$ \\
Initial visual acuity (logMAR) & $2.44 \pm 0.13$ & $0.14^{*}$ \\
Final visual acuity (logMAR) & $2.34 \pm 0.19$ & $0.10^{*}$ \\
\hline
\end{tabular}

Values are presented as number, number $(\%)$, or mean \pm standard deviation.

$\log \mathrm{MAR}=$ logarithm of the minimum angle of resolution.

${ }^{*}$ Student $t$-test; ${ }^{\dagger}$ Chi-square test. 
time, owing to the lack of randomization, allocation bias might also exist within each selected study.

\section{Retrospective analysis}

Pertinent demographic information, risk factors, as well as the type of supplementary treatments that were received for each group are listed in Table 2. In the HBOT group, mean elapsed time between symptom onset and diagnosis was $9.66 \pm 4.44$ hours, while the mean elapsed time between symptom onset and HBOT initiation was $18.27 \pm$ 10.4 hours. In the control group, mean elapsed time between symptom onset and diagnosis was $41.47 \pm 55.59$ hours. Hyperbaric treatment was stopped for three patients due to anxiety, asymptomatic bradycardia, and one patient that developed a tonic-clonic seizure. It is worth noting that the latter patient did not have an underlying seizure disorder, and further workup showed no underlying etiology for the seizure.

Analysis of our results demonstrates that there is no significant difference for initial, final, or change in VA of either group (Table 1). Likewise, incidence of NVG did not differ significantly between the treatment/HBOT (20.0\%) and no-HBOT/control groups $(15.2 \%)(p=0.676)$.

\section{Discussion}

Owing to large oxygen demand, the retina is highly susceptible to ischemia, with experimental animal studies in rhesus monkeys demonstrating an irreversible damage after 105 minutes versus good recovery with up to 97-98 minutes of ischemia [12]. To counter this visually debilitating effect of CRAO, many different treatment modalities are suggested, including HBOT. The rationale for application of HBOT is the dual vascular supply of retina, with the presumption that high oxygen circulation in the choroid might provide enough oxygen diffusion to the inner retinal layers to maintain viability [13]. Nevertheless, HBOT remains an area of controversy among many ophthalmologists and this controversy is once again surfaced in our study, where visual benefit was neither observed under our clinical settings nor in the meta-analysis.

Referring to the studies in rhesus monkeys [12], given the dramatic change of visual fate over a matter of few minutes time elapse, it appears that the source of bias and controversy among reported studies might stem from the timing of treatment. For instance, in comparison to our retrospective analysis with average time elapse of 18 hours from symptom onset to oxygen therapy, other studies that show improved VA from HBOT initiated treatment within 6 to 12 hours of visual loss $[9,14,15]$. Therefore, it is still possible that there might be beneficial effects from very early administration of HBOT. Indeed, of the three studies selected in our meta-analysis, the visual benefits became more evident with earlier treatments, where Rozenberg et al. [11] and Beiran et al. [9] report significant improvement in the final VA with time elapse of 9 and $<8$ hours, respectively. A similar conclusion is also drawn in a randomized controlled trial for CRAO patients undergoing intravenous tissue-type plasminogen activator, where despite overall negative results, subgroup analysis of data demonstrates beneficial visual outcome in patients who received intravenous tissue-type plasminogen activator within 6 hours of symptoms onset [5].

It is worth mentioning that while our meta-analysis and retrospective data did not show a beneficial effect from HBOT in the setting of CRAO, per prior studies, visual benefits might exist for a select number of patients that receive treatment in the very early course of disease [16]. This is not surprising since primate studies indicate that complete or almost total optic nerve atrophy and nerve fiber damage occurs after 240 minutes of central retinal arterial occlusion [17]. Nevertheless, given the potential time elapse until diagnosis as well as subsequent delay in the setup of HBOT, it is only feasible for a small portion of patients to receive treatment within early hours of symptoms onset. In fact, a recent study by Chan et al. [18] demonstrates that presentation of CRAO patients to emergency department is often delayed, resulting in limited application of acute treatments such as intravenous thrombolysis despite having a protocol in place [18]. Of note, among the patients presented to our tertiary care center, only three have received HBOT within 8 hours of symptoms' onset. In these patients, two have maintained stable VA of light perception and hand motion at long-term follow-up while the third patient VA decreased from hand motion to light perception.

In summary, while some individual studies conclude that early treatment of CRAO patients with HBOT improves the final VA, our meta-analysis as well as retrospective study fails to replicate a similar conclusion. Large, 
randomized studies are required to better understand the role of HBOT in treatment of CRAO but at the same time, strong considerations must also be made towards HBOT potential adverse effects including barotrauma, ear pain, tympanic membrane rupture, and generalized seizures due to the oxygen toxicity of the central nervous system [19].

\section{Conflicts of Interest: None.}

Acknowledgements: None.

Funding: None.

\section{References}

1. Mehta N, Marco RD, Goldhardt R, Modi Y. Central retinal artery occlusion: acute management and treatment. Curr Ophthalmol Rep 2017;5:149-59.

2. Farris W, Waymack JR. Central retinal artery occlusion [Internet]. Treasure Island: StatPearls Publishing; 2021 [cited 2021 Oct 20]. Available from: https://www.ncbi.nlm.nih. gov/books/NBK470354/.

3. Mouradian M, Wijman CA, Tomasian D, et al. Echocardiographic findings of patients with retinal ischemia or embolism. J Neuroimaging 2002;12:219-23.

4. Varma DD, Cugati S, Lee AW, Chen CS. A review of central retinal artery occlusion: clinical presentation and management. Eye (Lond) 2013;27:688-97.

5. Chen CS, Lee AW, Campbell B, et al. Efficacy of intravenous tissue-type plasminogen activator in central retinal artery occlusion: report from a randomized, controlled trial. Stroke 2011;42:2229-34.

6. Murphy-Lavoie H, Butler F, Hagan C. Central retinal artery occlusion treated with oxygen: a literature review and treatment algorithm. Undersea Hyperb Med 2012;39:94353.

7. Moher D, Liberati A, Tetzlaff J, et al. Preferred reporting items for systematic reviews and meta-analyses: the PRISMA statement. PLoS Med 2009;6:e1000097.

8. Navy Department. US Navy diving manual. Revision 6.
Washington, DC: Navy Sea Systems Command; 2011.

9. Beiran I, Goldenberg I, Adir Y, et al. Early hyperbaric oxygen therapy for retinal artery occlusion. Eur J Ophthalmol 2001;11:345-50.

10. Menzel-Severing J, Siekmann U, Weinberger A, et al. Early hyperbaric oxygen treatment for nonarteritic central retinal artery obstruction. Am J Ophthalmol 2012;153:454-9.

11. Rozenberg A, Hadad A, Peled A, et al. Hyperbaric oxygen treatment for non-arteritic central retinal artery occlusion retrospective comparative analysis from two tertiary medical centres. Eye (Lond) 2021 Jun 17 [Epub]. https://doi.org/ 10.1038/s41433-021-01617-8.

12. Hayreh SS, Weingeist TA. Experimental occlusion of the central artery of the retina. IV: retinal tolerance time to acute ischaemia. Br J Ophthalmol 1980;64:818-25.

13. Olson EA, Lentz K. Central retinal artery occlusion: a literature review and the rationale for hyperbaric oxygen therapy. Mo Med 2016;113:53-7.

14. Hadanny A, Maliar A, Fishlev G, et al. Reversibility of retinal ischemia due to central retinal artery occlusion by hyperbaric oxygen. Clin Ophthalmol 2016;11:115-25.

15. Lopes AS, Basto R, Henriques S, et al. Hyperbaric oxygen therapy in retinal arterial occlusion: epidemiology, clinical approach, and visual outcomes. Case Rep Ophthalmol Med 2019;2019:9765938.

16. Soares A, Gomes NL, Mendonca L, Ferreira C. The efficacy of hyperbaric oxygen therapy in the treatment of central retinal artery occlusion. BMJ Case Rep 2017;2017:bcr 2017220113.

17. Hayreh SS, Jonas JB. Optic disk and retinal nerve fiber layer damage after transient central retinal artery occlusion: an experimental study in rhesus monkeys. Am J Ophthalmol 2000;129:786-95.

18. Chan W, Flowers AM, Meyer BI, et al. Acute central retinal artery occlusion seen within 24 hours at a tertiary institution. J Stroke Cerebrovasc Dis 2021;30:105988.

19. Plafki C, Peters P, Almeling M, et al. Complications and side effects of hyperbaric oxygen therapy. Aviat Space Environ Med 2000;71:119-24. 